\title{
Experimental Optimization of growth Parameters of High Quality Green GaN Multiple Quantum Well by Metal-Organic Chemical Vapor Deposition
}

\author{
Feng Wen, Lirong Huang, Liangzhu Tong \& Deming Liu \\ Wuhan National Laboratory for Optoelectronics, College of Opto-electronics Science \& Engineering \\ Huazhong University of Sci. \& Technol, Wuhan 430074, China \\ E-mail:wenf2004@163.com
}

This work was supported by National Natural Science Foundation of China (no 60777019).

\begin{abstract}
The effects of grow parameters such as barrier thickness, flow ratio of group-III sources (TMIn/(TMIn+TMGa)), well temperature on the properties of green multiple quantum well (MQW) are investigated. The samples were grown by metal-organic chemical vapor deposition (MOCVD). High resolution x-ray diffraction (HRXRD) and room temperature photoluminescence (PL) were employed to analyze the results. The PL and HRXRD images show that a green MQW of high quality, at long wavelength $550 \mathrm{~nm}$, was obtained by controlling the GaN buffer quality, well growth temperature, and flow ratio of group-III sources (TMIn/(TMIn+TMGa)).
\end{abstract}

Keywords: GaN, Multi-quantum well (MQW), Metal-organic chemical vapor deposition (MOCVD)

\section{Introduction}

III-nitride semiconductors have been widely investigated for solid state lighting. It is promising due to $\mathrm{GaN}$, InN has a very wide direct energy band gap. As $x$ changing, the energy band gap of compound $\operatorname{In}_{x} \mathrm{Ga}_{1-x} \mathrm{~N}$ is possible to change from $1.95 \mathrm{ev}$ to $3.44 \mathrm{ev}$, which could cover the whole visible light range. Although the short wavelength light emitting diodes (LED), typically from $440 \mathrm{~nm}$ to $530 \mathrm{~nm}$, have already been commercially available, it is a far way to obtain longer wavelength light such as amber or red. The growth technology for long wavelength nitride based LED faces big challenges in that the $\mathrm{InN}$ and $\mathrm{GaN}$ are not very miscible due to that the high equilibrium vapor pressure of nitrogen over $\mathrm{InN}$ is much higher that GaN (N. Yoshimoto, 1991, pp.2251-2253). When the indium content is high, the indium aggregation phenomena (H. K. Cho, 2000, pp. 247-249) occurred and the V-defect (A.M. Yong, 2007, pp.4496-4510) appeared. So it is of great importance to optimize the growth parameters to elongate the emitting wavelength. There are some groups studying on effects of the growth parameters on the blue LED, such as barrier thickness (S.K. Shee, 2007, pp.373-377), growth pressure (T.K. Kim, 2007, pp.469-473), barrier growth temperature ramping (Y. Wang, 2007, pp.033509), growth interruption (H. K. Cho, 2001, pp.2594-2597). But for the growth of green LED, most of the studies focus on the growth rate, growth temperature and annealing of the P-GaN, there are not many studies on the multi-quantum well (MQW) of the green LEDs, especially at the long wavelength $550 \mathrm{~nm}$.

In this paper, a process to optimize the qualities of green $\mathrm{MQW}$ at wavelength $550 \mathrm{~nm}$ grown by metal-organic chemical vapor deposition (MOCVD) is present. Several samples were grown under different growth conditions. During the process, the effects of different parameters such as barrier thickness, well growth temperature, flow rate ratio of Group III source (TMIn/(TMIn+TMGa)) on the properties of the green MQW are studied and analyzed.

\section{Experiments}

Fig.1 is the schematic epitaxial structure of the green MQW, which was grown on a $\left(\begin{array}{llll}0 & 0 & 0 & 1\end{array}\right)$ sapphire substrate with a MOCVD, the Thomas Swan Close Coupled Showerhead Reactor $6 \times 6$ inch Systems. The whole growth process was monitoring by the real-time In-Situ Reflectance Monitor. Trimethylgallium (TMGa), trimethylindium (TMIn), and ammonia (NH3) were used as precursors for $\mathrm{Ga}$, In, and $\mathrm{N}$ elements, respectively. The carrier gas was hydrogen and the growth pressure was maintained at 400 mbar for the growth of MQW. Before growing, the substrates were treated by thermal baking at $1100^{\circ} \mathrm{C}$ in hydrogen to remove the surface contamination for half an hour. The growth process is procedure in the following procedures. After the deposition of the $30 \mathrm{~nm}$ low temperature nucleation layer at $530{ }^{\circ} \mathrm{C}$, a $1.5 \mu \mathrm{m}$ high temperature $\mathrm{GaN}$ was grown at $1050{ }^{\circ} \mathrm{C}$. Then five periods of quantum well were deposited. For every quantum well, a $2 \mathrm{~nm}$ cap layer grown 
at $900{ }^{\circ} \mathrm{C}$ was deposited to protect indium to escape when growing barrier. In order to analysis the effects of the growth parameters, the MQW of five different samples were grown in different parameters which are shown in Table 1 in detail. The well growth temperature for sample A, sample B, sample C, sample D, and sample $\mathrm{E}$ are $680{ }^{\circ} \mathrm{C}, 715{ }^{\circ} \mathrm{C}, 700{ }^{\circ} \mathrm{C}, 715^{\circ} \mathrm{C}, 725^{\circ} \mathrm{C}$, respectively. The barrier thicknesses for sample A, sample B, sample $\mathrm{C}$, sample D, and sample E are $20 \mathrm{~nm}, 20 \mathrm{~nm}, 20 \mathrm{~nm}, 15 \mathrm{~nm}, 15 \mathrm{~nm}$, respectively. The TMGa flow for all the samples is $4.91 \mathrm{~mol} / \mathrm{min}$. The TMIn flow rate is changed to increase the TMIn ratio in the group-III sources flow. The exact TMIn flow rate for sample A, sample B, sample C, sample D and sample E are $12.26 \mathrm{~mol} / \mathrm{min}, 12.5$ $\mathrm{mol} / \mathrm{min}, 12.5 \mathrm{~mol} / \mathrm{min}, 16.07 \mathrm{~mol} / \mathrm{min}$ and $17.86 \mathrm{~mol} / \mathrm{min}$, respectively. The flow ratio of group-III sources (TMIn/(TMIn+TMGa)) were calculated according to the TMIn flow rate and TMGa flow rate and the results for sample A, sample B, sample C, sample D, and sample E are $0.714,0.718,0.718,0.766,0.784$, respectively.

The crystal quality and performance of these samples were analyzed by high resolution X-ray diffraction (HRXRD) and room temperature photoluminescence (PL). The HRXRD image were made by Philips X-pert double crystal X-ray diffractometer with $\mathrm{Ge}(220)$ single crystal, operated at $40 \mathrm{kV}$ and $40 \mathrm{~mA}$. A He-Cd laser operated at $325 \mathrm{~nm}$ was used for room temperature PL measurement.

\section{Measurement results and discussions}

Fig.2 shows room temperature PL spectra of all the samples. The small multiple peaks in both experimental PL spectra of sample A and sample B are due to the Fabry-Perot effect (multi-wavelength interference between the $\mathrm{GaN} /$ air interface and GaN/sapphire interface). The different spaces between neighbor peaks indicate the different thicknesses of sample A and sample B. Fig.3 presents full-width at half-maximum (FWHM) of the HRXRD $\omega$ scans for sample B, sample C, sample D, sample E and sample H, from which we can evaluate the GaN buffer quality, the smaller, the better. Peak information is important to compare the properties of different samples. But it is not easy to find the emitting peak wavelength and peak intensity because of the interface effect. For this reason, Gauss fitting was used to obtain the peak information. Fig.4 shows the room temperature PL peak wavelength of all samples except sample A, which shows all the emitting wavelength are in the green light range. Fig.5 is the room temperature PL peak intensity of Gauss fitting curve for all the samples except sample A.

Normally, decreasing the well growth temperature is one way to elongate the emitting wavelength of the MQW. First we studied the relation between the growth temperature and quantum well quality. As we know, indium has a low melting point. If we grow $\mathrm{InGaN} \mathrm{QW}$ at low temperature, it is possible to reduce the volatilization of indium and increase the In composition in the QW thus lengthen the emitting wavelength. Therefore, sample A was first grown at a low temperature $680^{\circ} \mathrm{C}$ and another sample $\mathrm{B}$ was grown at a relative higher growth temperature $715^{\circ} \mathrm{C}$. In Fig.2, it shows that the emitting wavelength of sample B is longer than sample A, but the PL intensity of sample B is much higher than sample A. Fig.3 indicates that the FWHM of sample B is higher than that of sample A, which means the GaN buffer quality of sample B is worse than sample A. Normally, the LED grown on a better buffer will be better if the MQW were grown at the same condition. But PL intensity of sample B is much higher than that of sample A. This is believed to be due to too low growth temperature results in bad quantum well quality, which means decreasing the growth temperature is possible to obtain longer wavelength, but too low growth temperature would decrease the quality of the quantum well too.

Although the crystal quality of sample B is good, the PL wavelength of sample B is a little shorter. The QW growth temperature of sample $\mathrm{C}$ was slightly decreased to elongate the emitting wavelength. In the same time, we improved the grow quality of GaN buffer. Normally; the PL intensity should be decreased because too low growth temperature always results in a bad crystal quality for QW. But the PL intensity of sample C is higher than sample B as shown in Fig.2 and Fig.5. The increase of the PL intensity here is believed to be due to the growth temperature here is not too low and the increase of the buffer quality which we can see from Fig.3. PL results for sample $B$ and sample $C$ show that the buffer quality is very important too.

As stated before, growth temperature for QW and TMIn flow rate are two important parameters to affect the emitting wavelength. Low grow temperature could lengthen the emitting wavelength, but it also decrease the crystal quality too. Therefore, from sample D to sample E, the quantum well growth temperature and the flow ratio of group III sources (TMIn/(TMIn+TMGa)) are increased in the same time. As we know, thicker barrier thickness could enhance the emission efficiency. But too thick barrier thickness is possible to decrease the crystal quality too. For this reason, the barrier thickness of sample D and sample E were decreased compared to sample C. The PL peak wavelength $555 \mathrm{~nm}$ of sample D is longer than that of sample C which is $540 \mathrm{~nm}$, but the PL peak intensity of sample D is lower. The reason is that the GaN buffer quality of sample $\mathrm{C}$ is better as shown in Fig.3. When we increase the well growth temperature and TMIn flow rate again for sample E, the peak intensity 
is highly improved and the peak wavelength achieved at $560 \mathrm{~nm}$.

Fig.6 shows the 29/ $\omega$ HRXRD scans of the InGaN/ GaN MQW samples for the $\left(\begin{array}{llll}0 & 0 & 0 & 2\end{array}\right)$ reflection. The highest peak is the substrate peak. The satellite peaks around the substrate peak are due to the QW. It is obvious that the satellite peaks of sample D and sample E are much clear than sample C, partly in that the sample D and sample E has a smaller barrier thickness. In addition, the discernable satellite peak shows good growth interface between the quantum well and barrier in sample D and sample E too.

As shown in Fig.3, the FWHM of the HRXRD $\omega$ scans for sample $\mathrm{C}$ and sample $\mathrm{E}$ are nearly the same, which means sample $\mathrm{C}$ and sample $\mathrm{E}$ have similar buffer quality. But as shown in Fig.4 and Fig.5, light intensity of sample $\mathrm{E}$ is much higher than sample $\mathrm{C}$ and the emitting wavelength of sample $\mathrm{E}$ is higher than sample $\mathrm{C}$ too, despite sample $\mathrm{E}$ was grown at a high temperature. It indicates that it is much easier for indium to incorporate into InGaN at a high group III flow ratio. That is to say, it is better to keep a high group III flow rate ratio and growth the quantum well at a relative high temperature which could improve the crystal quality.

\section{Conclusions}

In summary, the effects of the MOCVD growth parameters such as the buffer quality, well growth temperature, barrier thickness, the flow ratio of group-III sources (TMIn/(TMIn+TMGa)) on properties of the green InGaN/GaN MQW were studied by PL spectrum and HRXRD. The experimental results show that good buffer quality will have a better emitting light intensity. Decreasing growth temperature or increasing the group III flow rate ratio could increase the indium incorporation, but too low temperature also lead to bad crystal quality thus decrease the emitting light intensity too. A high quality green MQW at wavelength $550 \mathrm{~nm}$ was obtained when keeping flow ratio of group-III sources (TMIn/(TMIn+TMGa)) at a high value and increasing the growth temperature as higher as possible in the same time.

\section{References}

N. Yoshimoto, T. Matsuoka, T. Sasaki, and A. Katsui. (1991). Photoluminescence of InGaN films grown at high temperature by metal-organic vapor phase epitaxy. Appl. Phys. Lett. Vol.59. pp.2251-2253.

H. K. Cho and J. Y. Lee. (2000). Superlattice-like stacking fault and phase separation of $\operatorname{In}_{x} \mathrm{Ga}_{1-x} \mathrm{~N}$ grown on sapphire substrate by metal-organic chemical vapor deposition. Appl. Phys. Lett. Vol. 77. pp. 247-249.

A.M. Yong, C.B. Soh, X.H. Zhang, S.Y. Chow, S.J. Chua. (2007). Investigation of V-defects formation in InGaN/GaN multiple quantum well grown on sapphire. Thin Solid Film, Vol.515. pp.4496-4510.

S.K. Shee, Y. Kwon, J. Lam, G. Gainer, G. Park, J.J. Song. (2000). MOCVD growth, stimulated emission and time-resolved PL studies of InGaN/(In)GaN MQWs: well and barrier thickness dependence. J. Cryst. Growth, Vol.221. pp. 373-377.

T.K. Kim, S.K. Shim, S.S. Yang, J.K. Son, Y.K. Hong and G.M. Yang. (2007).Influence of growth parameters on the properties of InGaN GaN multiple quantum well grown by metal-organic chemical vapor deposition. Current Applied Physics. vol.7. pp.469-473.

Y. Wang, X. J. Pei, Z. G. Xing, L. W. Guo, H. Q. Jia, H. Chen, J. M. Zhou. (2003). Effects of barrier growth temperature ramp-up time on the photoluminescence of InGaNGaN quantum wells. J. Apple. Phys., Vol.101.pp.033509.

H. K. Cho and J. Y. Lee. (2001). Effect of growth interruptions on the light emission and indium clustering of InGaNÕGaN multiple quantum wells. Appl. Phys. Lett. Vol.79. pp. 2594-2597.

Table 1. Detail sample growth parameters

\begin{tabular}{ccccccc}
\hline $\begin{array}{c}\text { Sample } \\
\text { ID }\end{array}$ & $\begin{array}{c}\text { Well } \\
\text { temprature }\end{array}$ & $\begin{array}{c}\text { Barrier } \\
\text { thickness }\end{array}$ & $\begin{array}{c}\mathrm{TMGa}_{2} \text { flow } \\
\text { in } \\
\text { well(mol/min) }\end{array}$ & $\begin{array}{c}\text { TMIn flow in } \\
\text { well(mol/min) }\end{array}$ & V/III(W) & TMIn/(TMIn+TMGa) \\
\hline $\mathrm{A}$ & $680^{\circ} \mathrm{C}$ & 17 & 4.91 & 12.26 & 23813 & 0.714 \\
$\mathrm{~B}$ & $715^{\circ} \mathrm{C}$ & 20 & 4.91 & 12.5 & 25645 & 0.718 \\
$\mathrm{C}$ & $700^{\circ} \mathrm{C}$ & 20 & 4.91 & 12.5 & 25645 & 0.718 \\
$\mathrm{D}$ & $715^{\circ} \mathrm{C}$ & 15 & 4.91 & 16.07 & 21279 & 0.766 \\
$\mathrm{E}$ & $725^{\circ} \mathrm{C}$ & 15 & 4.91 & 17.86 & 19610 & 0.784 \\
\hline
\end{tabular}




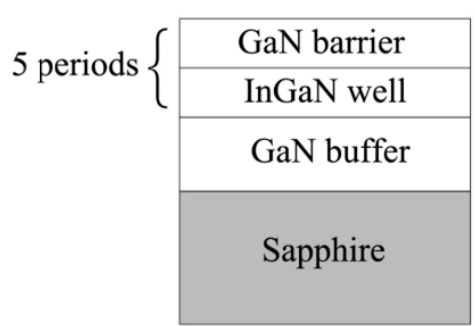

Figure 1. Schematic epitaxial structure

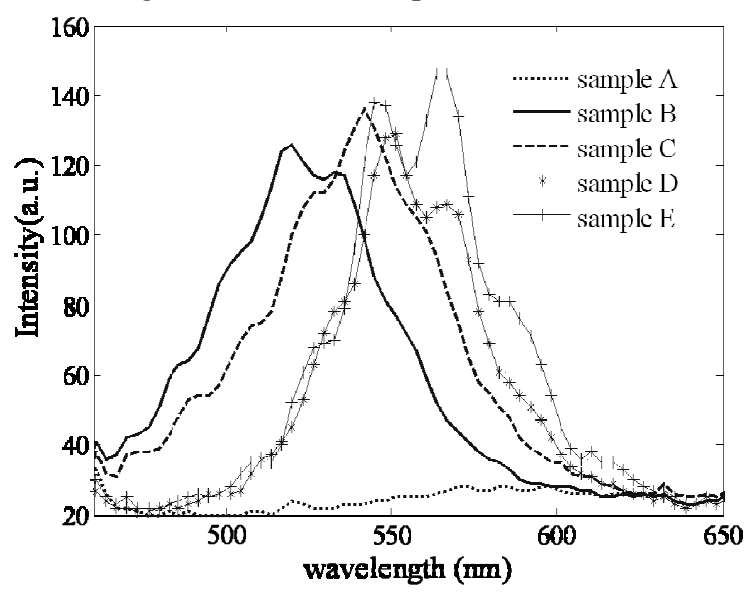

Figure 2. Room temperature PL spectra

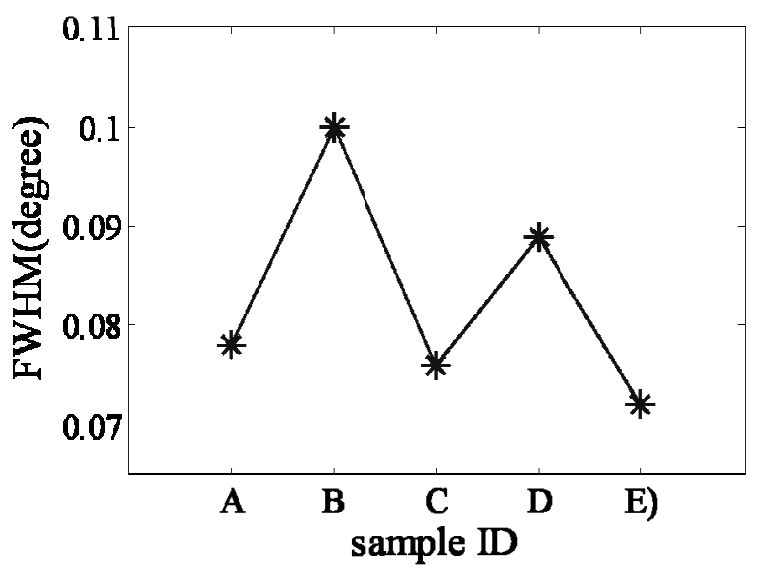

Figure 3. FWHM of HRXRD $\omega$ scans

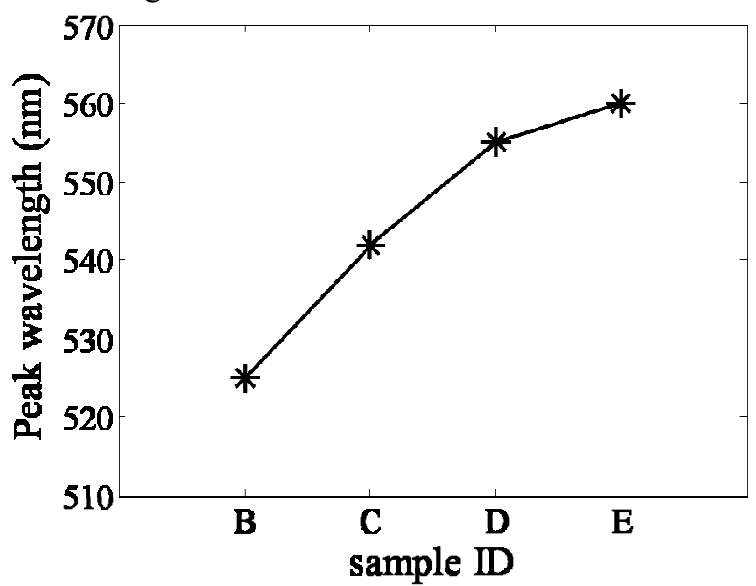

Figure 4. Peak wavelength of room temperature PL spectra 




Figure 5. Peak intensity of room temperature PL spectra

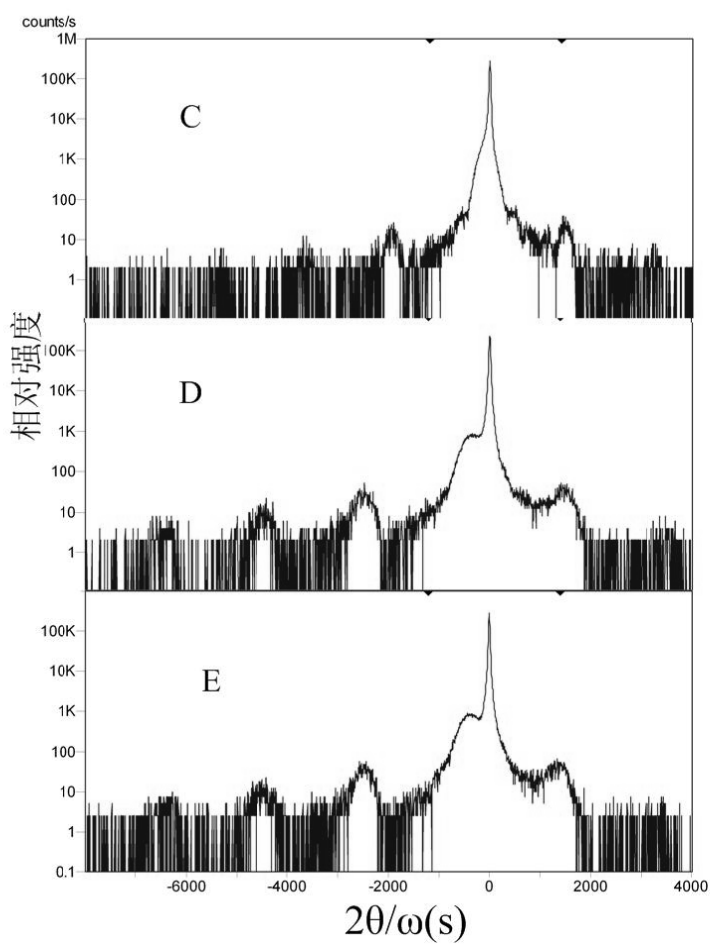

Figure 6. HRXRD 2 $29 / \omega$ scans for the $\left(\begin{array}{llll}0 & 0 & 0 & 2\end{array}\right)$ reflection from sample C, sample D and sample E 